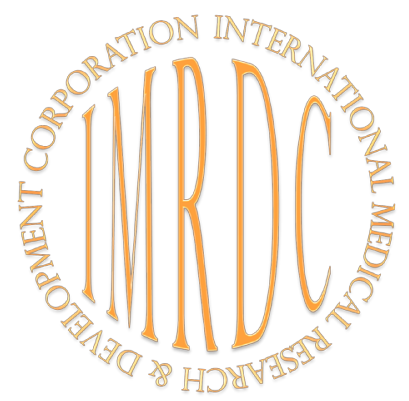

\title{
The Choice of Surgical Tactics for Appendicular Peritonitis in Children
}

\author{
Alexander A. Sleptsov ${ }^{1,2^{*}}$; Valentina A. Savina, $\mathrm{PhD}, \mathrm{ScD}^{1,2}$; Ahmed R. Varfolomeev, $\mathrm{PhD}^{1}$; \\ Valentin N. Nikolaev ${ }^{1}$; Eduard I. Petukhov ${ }^{2}$; Alexey L. Zuev'; Tumen E. Erdyneev²; \\ Sergey O. Kupryakov ${ }^{1,2}$; Vasily A. Grigoriev ${ }^{1,2}$; Olga S. Stepanova ${ }^{1,2}$; Evgeny A. Petrov ${ }^{1}$ \\ ${ }^{1}$ North-Eastern Federal University named after MK Ammosov, Yakutsk, Russia \\ ${ }^{2}$ Republican Hospital №1, National Centre of Medicine, Yakutsk, Russia
}

\begin{abstract}
This article presents the experience of using the modified Mannheim Peritonitis Index (MPI) score with an intraoperative assessment of the severity of peritonitis in children, taking into account the duration of the disease before surgery.

Materials and Methods: We analyzed the results of treatment of 186 children under age 16 who were operated on for appendicular peritonitis in the period between 2011 and 2018. The method of scoring for appendicular peritonitis index (API) consists in summing up the points reflecting the risk factors for the disease. API provides 2 criteria for the severity of peritonitis: the first degree - API $<10$ points, the second degree - API $\geq 10$ points. Ten or more points are an indication for laparotomy.

Results: With the definition of criteria for intraoperative assessment of the severity of peritonitis (API), the number of indications for laparotomy decreased. So, for the period from 2011 to 2014, the number of laparotomies performed with the subsequent programmed sanitation of the abdominal cavity was 17/14\% cases; from 2015 to 2018, the inflammatory process in the abdominal cavity were assessed with the determination of the intraoperative index of peritonitis severity - the number of conversions was 15/11\% cases. (International Journal of Biomedicine. 2019;9(2):134-138.)
\end{abstract}

Key Words: appendicular peritonitis $\bullet$ appendicular peritonitis index $\bullet$ Mannheim peritonitis index

\section{Abbreviations}

AP, appendicular peritonitis; API, appendicular peritonitis index; MPI, Mannheim peritonitis index

\section{Introduction}

Operations with acute appendicitis are the most frequently performed emergency operations on the abdominal organs and reach up to $70 \%$ of all abdominal interventions. ${ }^{(1,2)}$ Diffuse peritonitis in children is often a complication of destructive appendicitis. $^{(3)}$ In the treatment of diffuse peritonitis, the reduction of postoperative complications, ${ }^{(4-6)}$ including the frequency of repeated interventions and adhesive obstruction, is important. The choice of the optimal volume of surgery is influenced by intraoperative findings - the nature of the

*Corresponding author: Alexander A. Sleptsov, NorthEastern Federal University named after MK Ammosov, Yakutsk, Russia.E-mail: sashaogh@mail.ru effusion, fibrin, and the prevalence and presence of adhesions and abscesses. Also important is the duration of peritonitis before surgery, that is, the stage of peritonitis. ${ }^{(6)}$

In pediatric surgery, there are no clear criteria for determining the extent of the operation; typically, surgeons assess the severity of peritonitis. ${ }^{(7,8)}$ Laparotomy is the method of choice in most cases, and underestimating the severity of peritoneal inflammatory changes leads to repeated operations. ${ }^{(9)}$

To assess the severity of peritonitis and select the extent of surgical intervention, we proposed using an index. As the basis, we used the MPI. In 1987, a group of German surgeons in Mannheim developed the MPI to predict the outcome of purulent peritonitis in cancer patients. ${ }^{(10)}$ Subsequently, the MPI was used to assess the severity and prognosis of peritonitis outcome in patients with a general surgical profile. 
The calculation of the MPI includes 8 factors, each of which is scored from 0 to 12 . Quite quickly, the MPI received international recognition as an accurate and reliable assessment method with high sensitivity, from $83 \%$ to $98 \%$. $^{(11-13)}$

\section{Materials and Methods}

The study was performed in the Department of Pediatrics and Pediatric Surgery at North-Eastern Federal University named after MK Ammosov and in the Department of Purulent Surgery of the Pediatric Center of the Republican Hospital №1, National Centre of Medicine.

We analyzed the results of treatment of 186 children under age 16 who were operated on for AP in the period between 2011 and 2018. The diagnosis was confirmed by objective examination, radiographic examination of the abdominal organs, abdominal ultrasound examination, and laboratory data.

All patients (115/61.8\% boys and 76/38.2\% girls) were admitted to hospital for emergency reasons. The age of patients ranged from 4 months to 14 years (mean age of $8.2 \pm 3.7$ years). The terms of admission of patients into the hospital with signs and symptoms of AP are presented in Table 1. According to the classification of K. Simonyan, ${ }^{(14)}$ the distribution of patients was as follows: a reactive stage (the first 24 hours) $-88 / 47.3 \%$ patients, a toxic stage (24-72 hours) $-88 / 47.3 \%$ patients, and a terminal stage (over 72 hours) $-10 / 5.3 \%$ patients

Table 1.

The terms of admission of patients into the hospital with AP

\begin{tabular}{|l|c|c|}
\hline \multicolumn{1}{|c|}{ Disease duration } & Absolute & $\%$ \\
\hline Up to 1 day inclusive & 88 & $47.3 \%$ \\
\hline From 1 to 2 days & 63 & $33.8 \%$ \\
\hline From 2 to 3 days & 25 & $13.4 \%$ \\
\hline More than 3 days & 10 & $5.3 \%$ \\
\hline Total & 186 & $100 \%$ \\
\hline
\end{tabular}

Between 2011 and 2018, 1,868 children under age 16 were operated on for acute appendicitis and its complications. Until 2015, diagnostic laparoscopy was used in $95 \%$ of cases; appendectomy was performed endoscopically in $87 \%$ of cases. Since 2015, diagnostic laparoscopy has been used for all forms of appendicitis and its complications; conversions to open appendectomy were performed in 15 cases.

In $159 / 85.5 \%$ cases of peritonitis, the operation was started with diagnostic laparoscopy. During diagnostic laparoscopy, API was performed, taking into account the following factors:

1. Disease duration was considered, up to or over 72 hours.

2. The nature of effusion in the abdominal cavity during peritonitis is purulent or with the presence of fecal contents.

3. The nature of fibrin was assessed by its density and friability, the possibility of separation from the walls of the intestine and peritoneum.
4. The prevalence of peritonitis was assessed according to the anatomical regions of the abdominal cavity, with 5 or more areas affected; the prevalence of peritonitis was assessed by 2 points.

5. The presence of adhesions, their friability and density during separation.

6. The presence of inter-intestinal abscesses is a prognostic for a severe course of peritonitis, which indicates a violation of the primary immune response, and it suggests a risk of postoperative complications.

API provides 2 criteria for the severity of peritonitis: the first degree - API $<10$ points, the second degree - API $\geq 10$ points. The method of scoring for API consists in summing up the points reflecting the risk factors for the disease (Table 2). Ten or more points are an indication for laparotomy.

Table 2.

Appendicular peritonitis index

\begin{tabular}{|l|l|c|}
\hline \multicolumn{1}{|c|}{ Factor } & \multicolumn{1}{|c|}{ Severity } & Points \\
\hline \multirow{2}{*}{ Disease duration } & up to 72 hours & 1 \\
\cline { 2 - 3 } & over 72 hours & 3 \\
\hline \multirow{2}{*}{ Nature of effusion } & Purulent & 1 \\
\cline { 2 - 3 } & Fecal & 2 \\
\hline \multirow{2}{*}{ Nature of fibrin } & Loose & 1 \\
\hline \multirow{2}{*}{ Prevalence of peritonitis } & Dense & 2 \\
\hline \multirow{2}{*}{ The presence of adhesions } & Up to 4 areas & 1 \\
\cline { 2 - 3 } & 5 or more areas & 2 \\
\hline \multirow{2}{*}{$\begin{array}{l}\text { The presence of inter-intestinal } \\
\text { abscesses }\end{array}$} & Loose adhesions & 1 \\
\cline { 2 - 3 } & Dense adhesions & 2 \\
\hline & None & 1 \\
\cline { 2 - 3 } & Plural & 3 \\
\hline
\end{tabular}

When evaluating less than 10 points, it is recommended to continue the operation endoscopically. In the presence of inflammatory effusion, the primary sanitation of the abdominal cavity is carried out, which begins with the evacuation of purulent exudate from the focus area using an electric suction device. Then the coagulation of the appendix mesentery by a monopolar coagulator is performed; the monopolar coagulator of the "hook" type is used for the stump. On the basis of the stump of the appendix, Raeder's loop with a conductor is superimposed, intracorporeally tightened, and suture material vicryl $2 / 0$ or $3 / 0$ is used. The appendix is evacuated through a $10 \mathrm{~mm}$ or $12 \mathrm{~mm}$ trocar. If it is impossible to remove the appendix through the trocar, the evacuation of the latter is carried out in a container, with an extension of the wound. The final sanitation of the abdominal cavity is performed with an aqueous solution of $0.02 \%$ chlorhexidine with a revision of all areas and subphrenic spaces. In our cases, the abdominal cavity was drained according to A. Generalov, ${ }^{(15)}$ through the trocar installation site in $14 / 8.8 \%$ cases with marked inflammatory changes; a "cigar" tampon was installed locally in $58 / 36.5 \%$ cases. 
Complications of acute appendicitis were observed in 186 children: local peritonitis was detected in 98 cases, including appendicular infiltration in 1 case, periappendicular abscess - in 25 cases, and diffuse peritonitis - in 57 cases (Fig.1). In complicated appendicitis, drainage was performed; with severe "late" peritonitis, conversion was performed and programmed rehabilitation of the abdominal cavity was the treatment of choice.

Statistical analysis was performed using the Statistica 8.0 software package (StatSoft Inc, USA).
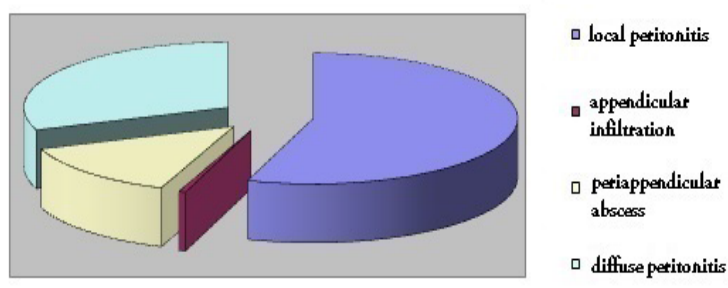

Fig. 1. Complications of acute appendicitis.

\section{Results}

$\mathrm{AP}$ at the beginning of the disease in the acute period is a controlled pathology in terms of surgical treatment. The volume of surgical treatment for peritonitis is limited by appendectomy and washing the abdominal cavity, and a drainage of pelvic cavity is performed according to indications. In our study, local peritonitis was detected in $4.89 \%$ of cases from performed appendectomies, and in the structure of peritonitis, it was $52.6 \%$.

In acute peritonitis with duration up to $48-72$ hours, an effusion into the abdominal cavity is more often exudativepurulent, as the response of the peritoneum to inflammation. Usually it is delimited by the small pelvis and the right iliac region. With a retrocecal, retroperitoneal location, periappendicular abscesses may form; abscesses also have a loose character without pronounced adhesions.

As a rule, when assessing the severity of peritonitis, the surgeon focuses on the presence of a purulent effusion in the abdominal cavity. Purulent effusion in the abdominal cavity up to 48-72 hours is of a liquid, exudative character, and its volume depends on the secretory features of each organism. In the study of exudate from the abdominal cavity in the first 48 hours from the onset of the disease, in most cases the microflora did not grow. Thus, in our opinion, the presence of a purulent effusion in the abdominal cavity, even in large quantities, is not an indication for conversion to open laparotomy.

In "late" peritonitis, with duration more than 48-72 hours, the nature of the effusion in the abdominal cavity is purulent and fecal, infiltrative-inflammatory changes in the peritoneum are expressed, and there is a spread of pus in almost all anatomical areas of the abdominal cavity. The resulting abscesses are dense, with thick fibrinous walls, with a pronounced adhesive process.

In the description of abscesses, we follow the classification of Doletsky et al. ${ }^{(8)}$ In Stages 1-2, disease duration up to 72 hours, local changes are characteristic of abscesses with loose walls; Stage 3 of abscess formation corresponds to the disease duration more than 72 hours - "late peritonitis." In our study, periappendicular abscesses $(n=25)$ made up $13.4 \%$ of the total number of complicated appendicitis, abscesses of Stages 1-2 were detected in $20 / 80 \%$ patients, abscesses of Stage 3 were in $5 / 20 \%$ children. In patients with periappendicular abscesses, the duration of the disease ranged from 2 to 12 days. Combined peritonitis is a combination of periappendicular abscess and free purulent effusion. This form is considered the outcome of an abscess; it is one of the most severe inflammatory processes, when infection can spread beyond the primary delimitation of purulent exudate. When determining the relationship between periappendicular abscesses and duration of disease, the correlation coefficient was $0.2(P=0.01)$.

We analyzed the effectiveness of methods for draining the abdominal cavity in children admitted with complications from different central district hospitals and treated in the Department of Purulent Surgery from 2006 to 2010. In most cases, the pelvic cavity was drained according to A. Generalov; ${ }^{(15)}$ since 2010, the method of choice for drainage in delimited peritonitis has been a "cigar" type tampon. Thus, over the past 8 years, the pelvic cavity drainage according to A. Generalov in AP was used in 10 cases, cigar-shaped drainage - in 59 cases. After pelvic cavity drainage according to A. Generalov ${ }^{(15)}$ complications $(n=4)$ in the form of pericultitis, pelvic abscess, stump failure, inter-intestinal abscess, and noncropped peritonitis were observed. After cigar-shaped drainage, complications were observed in 3 cases: perforation of the dome of the cecum and failure of the stump. Since some of the children arrived after an operation performed in the central district hospital with drains, it cannot be excluded that the method of installing the drains was not disturbed.

The volume of surgical treatment for peritonitis depends on the disease duration and the intraoperative picture, on the basis of which we identified the evaluation criteria.

Surgical tactics based on the results of diagnostic laparoscopy:

-In the presence of widespread pus, which has a liquid character, endoscopic tactics consisted of primary sanitation of the abdominal cavity, aspiration of a free purulent effusion, appendectomy, and drainage of the abdominal cavity.

-If there is a pronounced inflammatory infiltration of the tissues around the appendix, which create technical difficulties in excretion, it is necessary to switch to VolkovichDyakonov access, ${ }^{(16)}$ and finish the operation by draining the periappendicular abscess bed with cigar-shaped drainage.

-With pronounced inflammatory changes with a criterion for assessing the severity of peritonitis above 10 points, a laparotomy with a laparostomy is the method of choice for surgical treatment.

-In appendicular abscesses of Stages 2-3, the method of choice is mini-laparotomic access and cigar-shaped drainage of the cavity.

With the definition of criteria for intraoperative assessment of the severity of peritonitis, the number of indications for laparotomy decreased. So, for the period from 
2011 to 2014, the number of laparotomies performed with the subsequent programmed sanitation of the abdominal cavity was $17 / 14 \%$ cases; from 2015 to 2018 , the inflammatory process in the abdominal cavity were assessed with the determination of the intraoperative index of peritonitis severity - the number of conversions was $15 / 11 \%$ cases.

With the creation of criteria for intraoperative assessment of the severity of peritonitis, the number of indications for laparostomy has decreased. So, until 2014, the number of laparostomies was 15 cases, since 2015 - 13 cases; the frequency of programmed rehabilitation in patients has decreased. In total "late" peritonitis, endoscopic surgery is technically impossible. In patients with $\mathrm{API}<10$ points, the frequency of programmed sanitations decreased to 2 cases, which reduced the frequency of unreasonable laparotomy.

There is no marked change in the average number or frequency of laparostomies before the introduction and after the introduction of API, which indicates the validity of laparostomy. Median laparotomy with subsequent programmed sanitation of the abdominal cavity allows adequate intraoperative rehabilitation of the abdominal cavity and control of the course of the infectious process in the postoperative period. We adhere to the classical methodology of laparostomy proposed by N. Makoha. ${ }^{(9)}$

When comparing the average duration of the disease for patients with local and diffuse peritonitis in 2 independent samples, the differences in the average duration of the disease for patients with different types of peritonitis were statistically significant.

When determining the relationship between diffuse peritonitis and the duration of disease, the correlation coefficient was $0.32(P=0.001)$, with statistical significance. When analyzing the duration of inpatient treatment of children operated on for appendicular peritonitis, there was a decrease in the average number of bed-days (Fig.2).

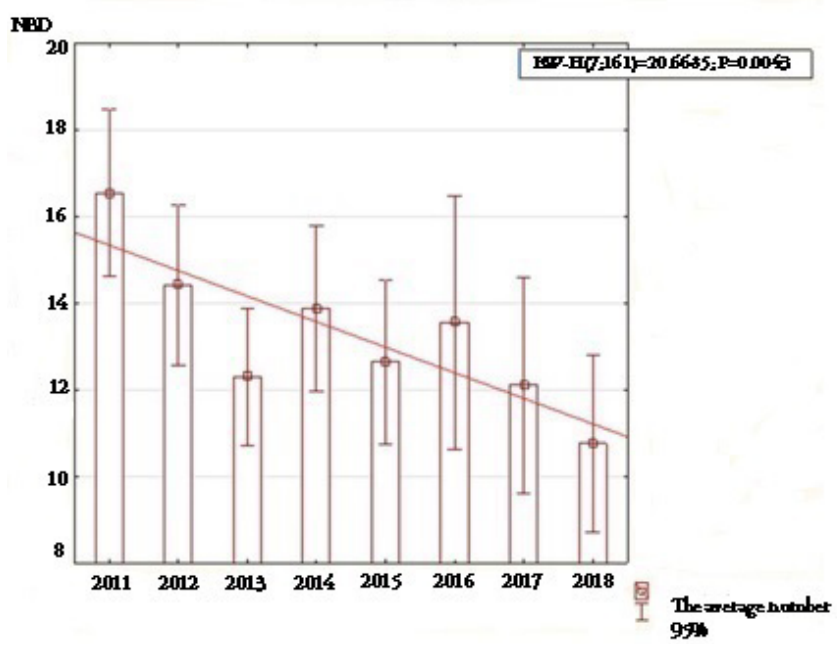

Fig. 2. The average number of bed-days (NBD) in the year.

\section{Conclusion}

With the introduction of the criteria for intraoperative assessment of the severity of peritonitis, the number of indications for laparotomy decreased $(P<0.05)$. So, for the period from 2011 to 2014 , the number of laparotomies performed with the subsequent programmed sanitation of the abdominal cavity was $17 / 14 \%$ cases; from 2015 to 2018 , the inflammatory process in the abdominal cavity was assessed to determine the intraoperative index of peritonitis severity - the number of conversions was $15 / 11 \%$ cases. Thus, not only the number of laparoscopy performed, but also the frequency of programmed sanitations in patients decreased. In total "late" peritonitis, endoscopic surgery is technically impossible. The frequency of programmed sanitations decreased to 2 cases in patients with $\mathrm{API}<10$ points, which reduced the frequency of unreasonable laparotomy.

\section{Competing Interests} interests.

The authors declare that they have no competing

\section{Acknowledgments}

We thank Sardana A. Ivanova for design assistance and translation of this manuscript.

\section{References}

1. Yudin YAB, Prokopenko YD, Fedorov KK, Gabinsky TA. Acute appendicitis in children. M.: Meditsina;1998. [In Russian].

2. Muehlstedt SG, Pham TQ, Schmeling DJ. The management of pediatric appendicitis: a survey of North American Pediatric Surgeons. J Pediatr Surg. 2004;39(6):8759; discussion 875-9.

3. Barskaja MA. Complex program of diagnosis and treatment of purulent surgical infection in children: Abstract of ScD Thesis. Ufa; 2000. [In Russian].

4. Dronov AF, Kotlobovsky VI, Poddubny IV. Laparoscopic surgery for acute appendicitis in children: real ways to improve the results of treatment. In: Proceedings of the symposium «Endoscopic surgery in children». Ufa; 2002:41-45. [In Russian].

5. Roshal' LM, Kapustin VA, Granikov OD, Karaseva OV. [Laparoscopic cleansing of the abdominal cavity in appendicular peritonitis in children]. Khirurgiia (Mosk). 1996;(4):35-7. [Article in Russian]

6. Tursunov KT, Ormantaev AK, Ruziddinov DB, Rakhmanov MA, Sagymbaeva AA. [Acute appendicitis and appendicular peritonitis]. Russian Journal of Pediatric Surgery, Anesthesia and Intensive Care. 2014;4(2):37-40. [Article in Russian].

7. Karaseva OV, Kapustin VA, Bryantsev AV. [Laparoscopic surgery for abscess forms of appendicular peritonitis in children]. Pediatric Surgery. 2005;3:25-29. [Article in Russian]. 8. Doletsky SYa, Shchitinin VE, Arapova AV. Complicated appendicitis in children. M.: Meditsina; 1982. [In Russian]. 9. Makokha NS. Treatment of severe forms of diffuse purulent peritonitis by the open method. In: Materials of the X Plenum Scientific Society of Surgeons of the Ukrainian SSR. Kiev; 1967:116-117. [In Russian].

10. Linder MM, Wacha H, Feldmann U, Wesch G, Streifensand RA, Gundlach E. [The Mannheim peritonitis index. An instrument for the intraoperative prognosis of 
peritonitis]. Chirurg. 1987;58(2):84-92. [Article in German]. 11. KrylovNN, Babkin OV,Babkin DO. [Mannheim peritonitis index, criteria for the selection of surgical intervention for perforated duodenal ulcer]. Surgery. 2016;7:18-22. [Article in Russian].

12. Sivaprahasam N, Vijayalakshmi S. Predictive value of mannheim peritonitis index (MPI) for mortality in perforated peptic ulcer. International Journal of Emerging Trends in Science and Technology. 2014;12:3:566-568.

13. Tushar D, Ramachandra L, Nair R, Digvijoy Sh. Evaluation of prognosis in patients, with perforation peritonitis using Mannheim's peritonitis index. International Journal of Scientific and Research Publication. 2015;5:2250-3153.

14. Simonyan KS. Peritonitis. M.: Meditsina; 1971. [In Russian]. 15. Generalov AI, Kovalev VI, Baurova LV. [Abdominal cavity drainage in children with appendicular peritonitis (a review of the literature)]. Vopr Okhr Materin Det. 1977;22(12):16-22. [Article in Russian].

16. Anisimov AIu, Podshivalov AG. [Method for creating approach by Volkovich-D'iakonov in technically complicated appendectomies]. Khirurgiia. (Mosk). 1999;(12):19-20.[Article in Russian]. 